\title{
Obtaining a 3D extension of Pascal theorem for non-degenerated quadrics and its complete configuration with the aid of a computer algebra system
}

\author{
Eugenio Roanes-Macías, Eugenio Roanes-Lozano \\ and Julio Fernández-Biarge
}

\begin{abstract}
Pascal's classic theorem states that: "the three intersection points of opposite sides of a closed hexagonal line, inscribed in a nondegerated conic, are collinear". The following extension of Pascal theorem to 3D is considered: "given a closed decagonal line, inscribed in a nondegerated quadric, whose opposite side-lines are secant, the five intersection points of opposite side-lines are coplanary". (A polygonal line with 10 sides is considered, because $10-1$ points determine a quadric, as $6-1$ points determine a conic). Obviously, in this extension to 3D of Pascal theorem some vertices of the polygonal line can not be freely chosen, but an interesting property has been found: the five diagonal lines passing through opposite vertices share a point. This property leads to a simple method to generate the configuration. Moreover, conditions of existence of this configuration are determined and the so called complete configuration is also described in detail. As large expressions appear when coordinates are used, we have developed a package on a computer algebra system that helps us to find and to automatically generate this configuration.
\end{abstract}

\section{Obtención de una extensión a 3D del teorema de Pascal para cuádricas no degeneradas y su configuración completa con la ayuda de un sistema de cómputo algebraico}

\begin{abstract}
Resumen. El teorema clásico de Pascal afirma: "los tres puntos de intersección de lados opuestos de una línea hexagonal cerrada, inscrita en una cónica no degenerada, son colineales". Se considera la siguiente extensión a 3D del teorema de Pascal: "dada una línea decagonal cerrada, inscrita en una cuádrica no degenerada, cuyos lados opuestos sean secantes, los cinco puntos de intersección de lados opuestos son coplanarios". (Se considera una línea poligonal de 10 lados, porque $10-1$ puntos determinan una cuádrica, del mismo modo que $6-1$ puntos determinan una cónica). Obviamente, en esta extensión a 3D del teorema de Pascal algunos vértices de la línea poligonal no pueden ser libremente elegidos, pero se ha encontrado una propiedad interesante: las cinco líneas diagonales que pasan por vértices opuestos de la poligonal son concurrentes en un punto. Esta propiedad conduce a un método sencillo para generar la configuración. Aun más, se determinan las condiciones de existencia de la configuración y se describe en detalle su configuración completa. Como al introducir coordenadas aparecen expresiones extensas, se ha desarrollado un paquete sobre un sistema de cómputo algebraico, que ayuda a determinar la configuración y a generarla automáticamente.
\end{abstract}

Presentado por / Submitted by José Javier Etayo Miqueo.

Recibido / Received: 21 de enero de 2009. Aceptado / Accepted: 4 de febrero de 2009.

Palabras clave / Keywords: Configuration theorems. Computer-aided theorem proving and discovery.

Mathematics Subject Classifications: 51A20, $68 \mathrm{U} 99$.

(C) 2009 Real Academia de Ciencias, España. 
"Mathematics is not a deductive science. When you try to [solve a problem] .... what you do is trial and error, experimentation, guesswork.

You want to find out what the facts are, and what you do is in that respect similar to what a laboratory technician does, but it is different in its degree of precision and information."

Paul Halmos

\section{Introduction}

We have been working for some time in the extension of classic geometric theorems to 3D using computational methods $[7,8,9,10,11,12,13,14]$.

In Section 4 of [13] and, more deeply, in [14], we had been able to extend Pappus 2D theorem classic configuration to 3D. As this configuration can be considered as a particular case of that of Pascal 2D theorem (when the conic degenerates into two lines), it is natural to consider as the next step the 3D extension of Pascal 2D theorem classic configuration. Such is the goal of the present work.

\subsection{Pascal 2D classic theorem}

Pascal 2D theorem classic configuration can be found in any standard geometry textbook. Let us begin revisiting it, trying to extract ideas about how to extend this theorem to 3D.

Given a conic $c$ (for instance, an ellipse) and a sequence of six points lying on $c: A_{1}, A_{2}, A_{3}, A_{4}$, $A_{5}, A_{6}$, (all different two by two), the closed polygonal which vertices are those points is considered. Now, denoting the sides of the polygonal the following way: $L_{1}=A_{1} A_{2}, L_{2}=A_{2} A_{3}, L_{3}=A_{3} A_{4}$, $L_{4}=A_{4} A_{5}, L_{5}=A_{5} A_{6}, L_{6}=A_{6} A_{1}$, if each side intersects it opposite one, then those three intersection points, $P_{1}=L_{1} \cap L_{4}, P_{2}=L_{2} \cap L_{5}, P_{3}=L_{3} \cap L_{6}$, are collinear (see Figure 1). This result is known as Pascal (classic) theorem.

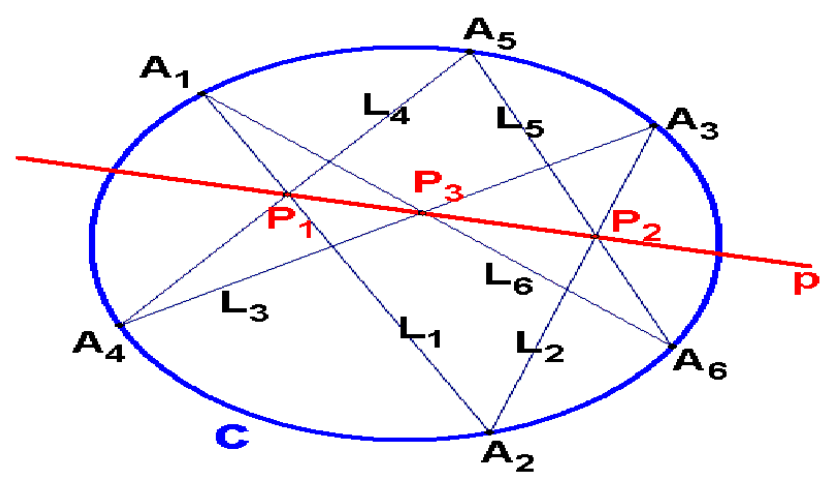

Figure 1. Pascal 2D classic configuration.

As the opposite sides (segments) could not intersect (i.e., could be disjoint), it is advisable to substitute the "sides" of the polygonal by "side-lines". In case two opposite side-lines are parallel, that they intersect at their point at infinity can be considered, and therefore it is advisable to consider this configuration in the projective plane. In this space, the result can be enunciated as follows: the opposite side-lines of a 
hexagonal closed polygonal inscribed in a conic intersect at collinear points (see Figure 2). The line, $p$, passing through those three points, is denoted Pascal line.

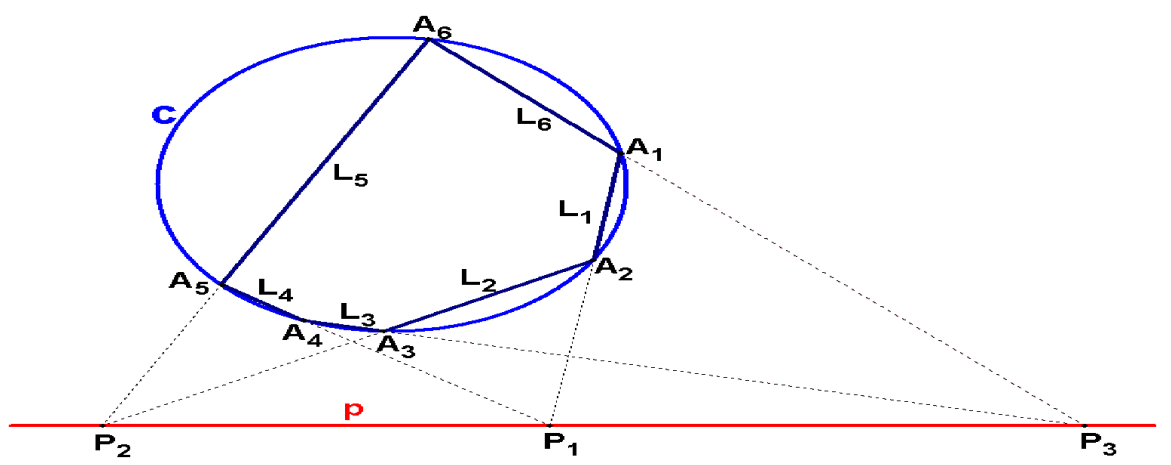

Figure 2. Configuration with intersecting side-lines.

\subsection{Determining Pascal 2D configuration}

Summarizing, Pascal classic configuration involves the following elements: the conic $c$; the six vertices of the closed inscribed polygonal, $A_{1}, A_{2}, A_{3}, A_{4}, A_{5}, A_{6}$; the six side-lines of the polygonal, $L_{1}, L_{2}, L_{3}, L_{4}$, $L_{5}, L_{6}$; the three intersection points of opposite side-lines, $P_{1}, P_{2}, P_{3}$; and Pascal line $p$, passing through the latter points.

The anomalous cases when two vertices are coincident or when the Pascal line passes through a vertex, are excluded. Therefore, the six vertices of the polygonal will be supposed to be different two by two and the Pascal line will be supposed not to pass through any vertex of the polygonal.

As a conic is determined by five points in the plane, Pascal classic configuration is determined by five vertices of the polygonal and by a sixth vertex on the conic passing through the other five points.

The following proposition details another way to determine the configuration, that will be useful to extend the problem to 3D.

Proposition 1 Pascal classic configuration is also determined by conic $c$, the four first vertices $A_{1}, A_{2}$, $A_{3}, A_{4}$ of the polygonal line and Pascal line $p$.

Proof. With the notation used above, from the given elements we can successively determine: $L_{1}=$ $A_{1} A_{2}, L_{2}=A_{2} A_{3}, L_{3}=A_{3} A_{4}, P_{1}=L_{1} \cap p, L_{4}=A_{4} P_{1}, A_{5}=L_{4} \cap c\left(A_{5} \neq A_{4}\right), P_{2}=L_{2} \cap p$, $L_{5}=A_{5} P_{2}, A_{6}=L_{5} \cap c\left(A_{6} \neq A_{5}\right), L_{6}=A_{6} A_{1}, P_{3}=L_{3} \cap p$, where only that $P_{3}$ lies on $L_{6}$ is left, but this membership relation holds as a consequence of Pascal theorem.

Remark 1 Obviously, in the previous Proposition 1 the four first vertices can be substituted by the three first sides $L_{1}, L_{2}, L_{3}$.

\subsection{The state of Pascal theorem extension to 3D}

The classic theorem due to Pascal (1623-1662) has already been extended in different ways.

Its extension to plane curves of degree greater than 2 was initiated by Clifford [3] and later developed by C. Fox [6] in different ways: 1) substituting the conic by a plane cubic and the hexagon by an octogon; 2) substituting the conic by a plane quartic and the hexagon by an decagon;3) proving that it is not possible to extend the result to algebraic curves of degree greater than 4 . 
Another way to extend Pascal classic 2D theorem appears in page 143 of section 144b of George Salmon treatise [15]. The 3D extension consists on substituting conic by ruled quadric and the six sides of the hexagon by two sets of three planes. If each of the planes of these sets intersects two of the planes in the other set in lines contained in the quadric, then the intersections of those planes with the third planes in the other set are coplanary lines. An analogous result, following a simpler and more intuitive way, has recently been obtained by one of the coauthors of this article [5]. It is the most modern result we know regarding the 3D extension of Pascal theorem, but it does not treat polygons inscribed in a quadric, but polygons whose side-lines are generatrices of the quadric (that is consequently a ruled quadric).

Another extension of Pascal 2D theorem was enunciated (without proof) by M. Chasles [2], proven by G. Salmon in section 144b (pages 141-142) of its treatise [15] and rediscovered by N. A. Court [4]. It can be enunciated this way: given a tetrahedron of vertices $V_{i} ; i=1,2,3,4$ and four planes, $\alpha_{i} ; i=1,2,3,4$, for each plane $\alpha_{i}$, the three intersection points with the three side-lines of the tetrahedron passing through vertex $V_{i}$ (that is, the three points $A_{i j}=\alpha_{i} \cap V_{i} V_{j} ; j \neq i$ ) and line $\rho_{i}$, intersection of $\alpha_{i}$ with the face-plane of the tetrahedron opposite to vertex $V_{i}$, are considered. Then, the twelve points $A_{i j}$ lie on a certain quadric if the four lines $\rho_{i}$ are coplanary. This 3D result was later extend to dimension $n$ by O. Bottema [1].

The work below treats the 3D extension of Pascal theorem to any non-degenerated quadric (needless to be ruled).

The case when the quadrics degenerate into a pair of planes (then Pascal 3D theorem turns into Pappus 3D theorem) has already been studied by this authors [14].

For our approach to Pascal 3D configuration, the converse of Pascal 2D theorem is of special interest. It can be enunciated the following way: given a conic, $c$, and a coplanar line, $p$, it is always possible to inscribe an hexagon in $c$ whose Pascal line is $p$. The proof of this result can be found in the article by C. Fox mentioned above [6] and will be used constructively in this article.

Summarizing, as far as we know, the 3D extension of Pascal 2D theorem treated in this article has nothing to do with those extensions previously developed.

\section{Our 3D extension of Pascal configuration}

When trying to extend to 3D Pascal classic configuration, it seems natural to substitute "conic" by "quadric". It also seems natural to substitute the six sides closed polygonal by a ten sides one, as $10-1$ points determine a quadric (the same way as $6-1$ points determine a conic).

On the other hand, in the projective plane two different lines always share a common point, meanwhile in the 3D projective space two different line can be disjoint (i.e., without common points). Consequently, when trying to extend to 3D Pascal classic configuration, that each pair of opposite sides of the ten sides polygonal share a common point has to be firstly required.

Finally, it seems natural to consider as thesis that these five intersection points of opposite sides of the polygonal are coplanary (the same way as the three intersection points of opposite sides of the polygonal are collinear in the $2 \mathrm{D}$ case).

These considerations lead to the following definition.

Definition 1 In the real projective $3 D$ space, a non-degenerated quadric, $S$, and a ten vertices $\left(A_{1}, A_{2}\right.$, $\ldots, A_{10}$ ) closed polygonal, which side lines are denoted $L_{1}=A_{1} A_{2}, L_{2}=A_{2} A_{3}, L_{3}=A_{3} A_{4}, \ldots$, $L_{9}=A_{9} A_{10}, L_{10}=A_{10} A_{11}$ are considered (as the polygonal is closed, $A_{11}=A_{1}$, what shortens notation). If all the following conditions are verified:

1) the polygonal is inscribed in the quadric, that is, its vertices verify $A_{i} \in S ; i=1, \ldots, 10$,

2) all vertices are different two by two, that is, $A_{i} \neq A_{j}$; for $i \neq j$,

3) each side-line and its opposite one, $L_{i}$ and $L_{i+5}$, do intersect, for $i=1, \ldots, 5$,

4) each plane containing a side-line and its opposite, $L_{i}$ and $L_{i+5}$, is different from the plane containing the following side-line and its opposite, $L_{i+1}$ and $L_{i+6} ; i=1, \ldots, 5$, (where $L_{11}$ is $L_{1}$ ), 
5) the five intersection points of opposite side-lines, denoted $P_{1}=L_{1} \cap L_{6}, P_{2}=L_{2} \cap L_{7}, P_{3}=L_{3} \cap L_{8}$, $P_{4}=L_{4} \cap L_{9}, P_{5}=L_{5} \cap L_{10}$, are coplanary,

then we shall say that these geometric elements constitute a Pascal 3D configuration. The plane through the five points $P_{1}, P_{2}, P_{3}, P_{4}, P_{5}$ will be named "Pascal plane of the configuration" and will be denoted $\pi$ (see Figure 3).

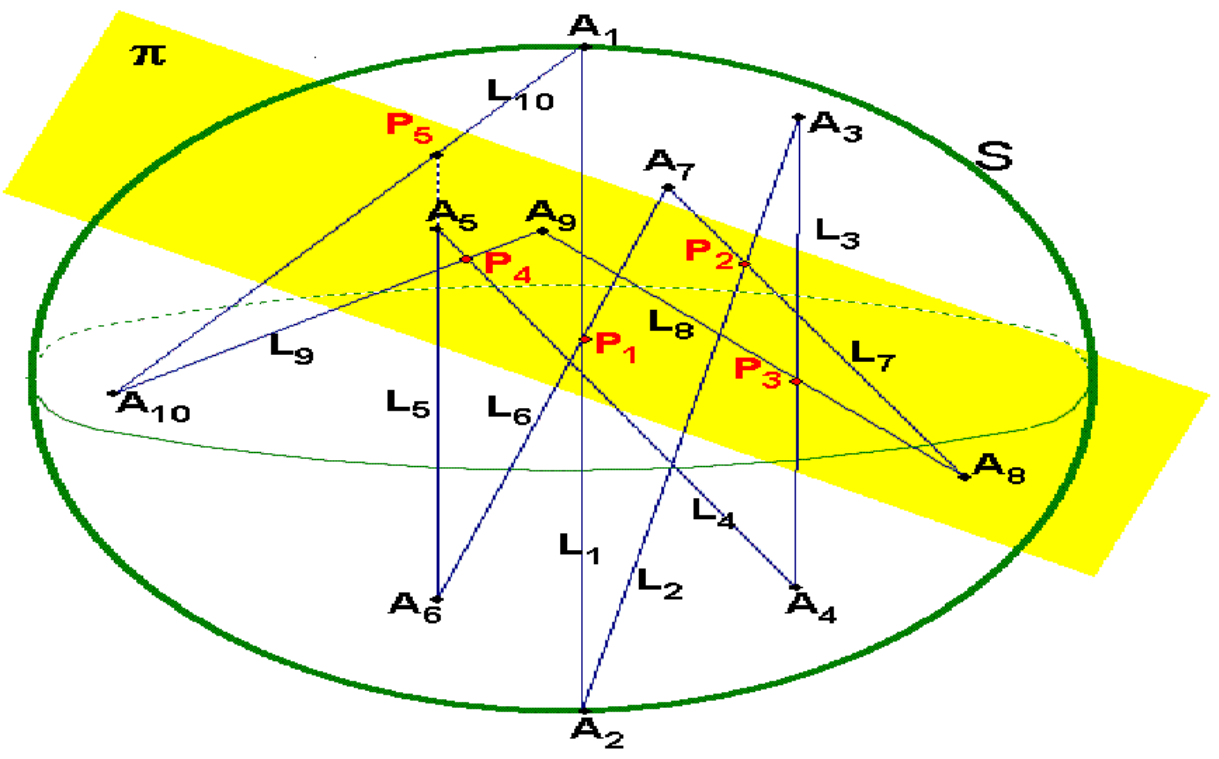

Figure 3. Pascal 3D configuration.

Now two questions arise: do such configurations exist?; and, in case the answer to the previous question is affirmative: how to construct them?

We shall begin by showing two very simple example, that will later bring ideas about how to construct Pascal 3D configurations.

\subsection{Configuration obtained from a pentagonal prism inscribed in a sphere}

Given a pentagonal prism inscribed in a spherical surface, the closed polygonal, which sides are the diagonals of the faces of the prism, is considered (Figure 4).

More precisely, given the spherical surface, $S$, with equation $x_{1}^{2}+x_{2}^{2}+x_{3}^{2}-3 x_{0}^{2}=0$, the closed polygonal line which vertices are $A_{1}=(1,1,-1,1), A_{2}=(1,1,1,-1), A_{3}=(1,-1,1,1), A_{4}=$ $(1,0,-\sqrt{(2)},-1), A_{5}=(1,-1,-1,1), A_{6}=(1,1,-1,-1), A_{7}=(1,1,1,1), A_{8}=(1,-1,1,-1)$, $A_{9}=(1,0,-\sqrt{(2)}, 1), A_{10}=(1,-1,-1,-1)$, different two by two and lying on $S$, is considered.

Its ten side-lines also satisfy conditions 3$)$ and 4$)$ of Definition 1 . The intersection points of opposite side-lines turn out to be: $P_{1}=(1,1,0,0), P_{2}=(1,0,1,0), P_{3}=(-2,1,-1+\sqrt{(2)}, 0), P_{4}=(-2,1,1+$ $\sqrt{(2), 0), P_{5}}=(-1,0,1,0)$.

The Pascal plane, $\pi$, passing through $P_{1}, P_{2}, P_{3}, P_{4}$ and $P_{5}$ turns out to be $x_{3}=0$ (as shown in Figure 4). Therefore this is a Pascal 3D configuration in the sense of Definition 1. 


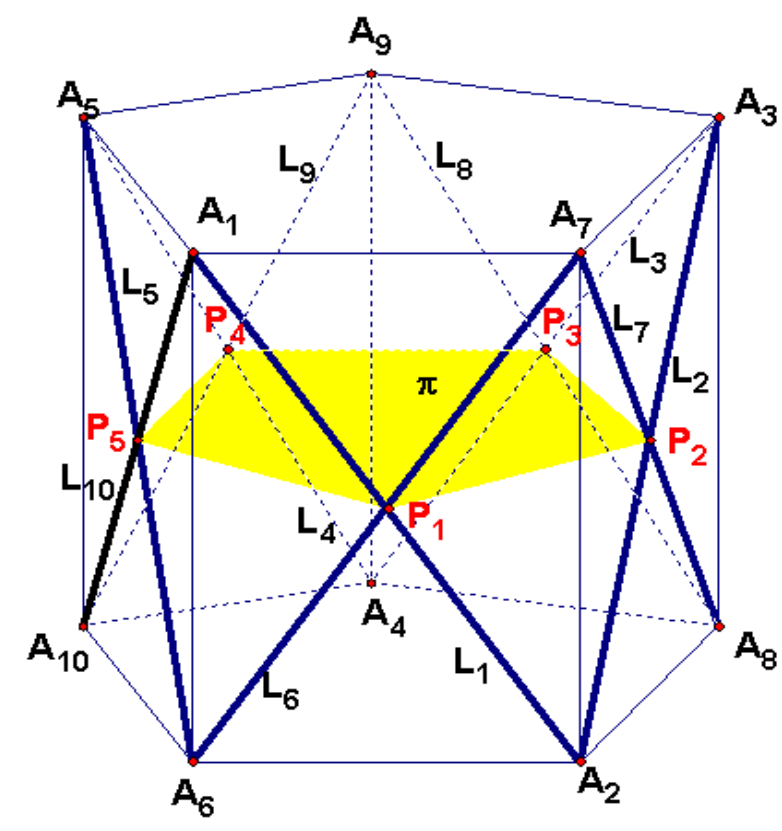

Figure 4. Configuration obtained from a pentagonal prism

\subsection{Pascal 3D configuration with centre of symmetry}

Given a polyhedron consisting of a cube with two opposite side faces pointed, inscribed in a spherical surface, $S$, the decagon of Figure 5, which sides are edges of this polyhedron and that admits as centre of symmetry the centre, $O$ of the cube, is considered.

More precisely, given the spherical surface, $S$, with equation $\left(x_{1}-1\right)^{2}+\left(x_{2}-1\right)^{2}+\left(x_{3}-1\right)^{2}-3 x_{0}^{2}=$ 0 , the closed polygonal line which vertices are $A_{1}=(1,2,0,0), A_{2}=(1,2,2,0), A_{3}=(1,2,0,2)$, $A_{4}=(1,1,1,1+\sqrt{(3)}), A_{5}=(1,2,2,2), A_{6}=(1,0,2,2), A_{7}=(1,0,0,2), A_{8}=(1,0,2,0)$, $A_{9}=(1,1,1,1-\sqrt{(3)}), A_{10}=(1,0,0,0)$, different two by two and lying on $S$, is considered.

Its ten side-lines also satisfy conditions 3$)$ and 4) of Definition 1 . The intersection points of opposite side-lines turn out to be: $P_{1}=(0,0,1,0), P_{2}=(0,0,-1,1), P_{3}=(0,-1,1,-1+\sqrt{(3)})$, $P_{4}=(0,1,1,1-\sqrt{3}), P_{5}=(0,1,0,0)$.

Let us observe that, as the polygonal admits a centre of symmetry, each side-line is parallel to its opposite one, and therefore they do intersect at their point at infinity. Consequently, the five intersection points of opposite side-lines lie on the plane at infinity, $x_{0}=0$. Therefore, this is a Pascal 3D configuration in the sense of Definition 1, and $x_{0}=0$ is its Pascal plane.

\section{Package for calculations automation}

Extraordinary long expressions arise when trying to analytically calculate the coordinates and equations of the elements of the configuration in not so simple cases. This fact makes it advisory to use a computational system enabling non-assigned variable handling and exact arithmetic.

We have used the paramGeo3D package, implemented in the computer algebra system Maple, that we had developed and implemented for the work described in [13]. It has been complemented with other commands and procedures that are useful for the computations that appear in the present work. We detail 


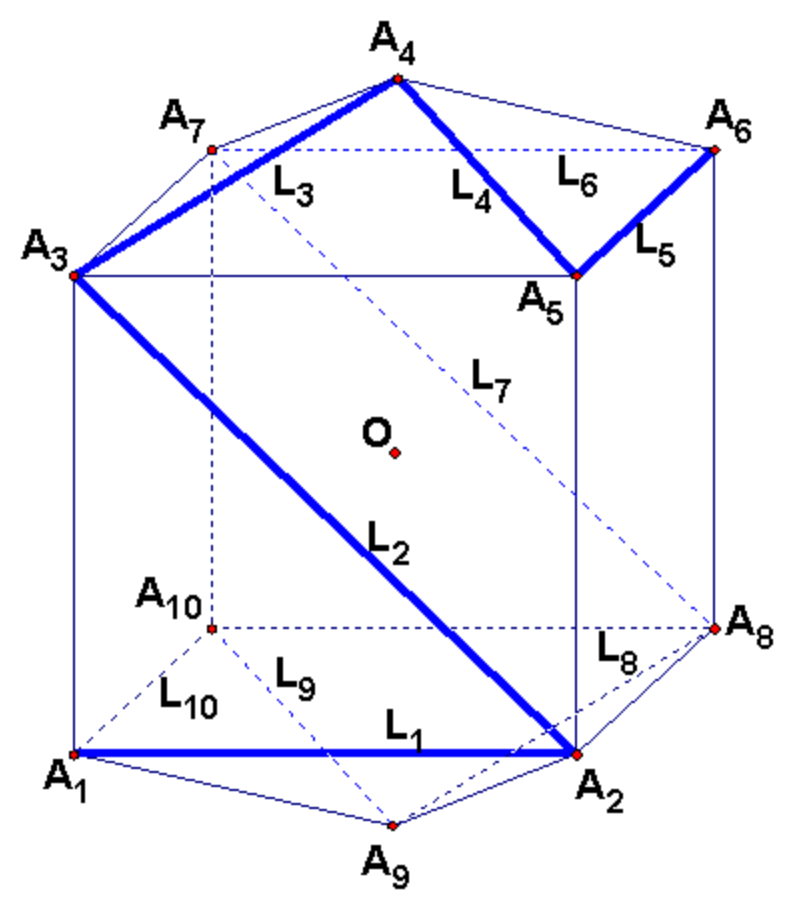

Figure 5. Configuration with centre of symmetry.

below those most frequently used.

- point (A) returns the list $\left[a_{0}, a_{1}, a_{2}, a_{3}\right]$, which elements are the projective coordinates of the point $A=\left(a_{0}, a_{1}, a_{2}, a_{3}\right)$.

- plane $(\mathrm{A}, \mathrm{B}, \mathrm{C})$ returns the equation of the plane through the three points $A, B, C$, that are supposed to be non collinear (it returns an error message in case they are collinear).

- line $(\mathrm{A}, \mathrm{B})$ returns the list of equations of two planes through points $A$ and $B$, that are supposed to be different (it returns an error message if the points are coincident).

- pointontine $(\mathrm{A}, \mathrm{B}, \mathrm{r})$, returns the point $P$ on the line through points $A$ and $B$, such that $\overrightarrow{A P}=$ $r \cdot \overrightarrow{A B}$, where $r$ is a real number or $\infty$.

- sphere returns the equation of the corresponding spherical surface, when applied (indistinctly) to its center and a point on it, or to its center and the radius, or also to four non-coplanary points.

- quadric returns the equation of the corresponding quadric, when applied to nine points that define it.

- intersection when applied to two previously defined algebraic varieties, which sum of degrees is $\leq 3$, returns their intersection points.

- vertex (A, S) returns the list $\left[a_{0}, a_{1}, a_{2}, a_{3}\right]$ of projective coordinates of point $A=\left(a_{0}, a_{1}, a_{2}, a_{3}\right)$ and adds list $\left[a_{0}, a_{1}, a_{2}, a_{3}\right]$ to the list $V E R T I C E S$, in case $A$ is on quadric $S$ and it is not yet in such list (it returns an error message in other case).

- newVertex $(r, \mathrm{~S})$ returns the intersection point of line $r$ with quadric $S$, not contained in list $V E R T I C E S$, and adds this point to such list. 
- isIn $(\mathrm{A}, \mathrm{Ob})$ returns truelfalse, depending on whether point $A$ is on object $O b$ (line, plane or quadric) or not.

- isPlaced $(\mathrm{A}, \mathrm{Ob})$ returns the result of substituting the coordinates of point $A$ in the equation or equations of object $O b$ (line, plane or quadric).

- equalpoints (A, B) returns true/false, depending on whether $A$ and $B$ are projective coordinates of the same point or of different points.

- equalVertices (L) returns the positions of the pairs of equal points in the list of vertices $L$.

- coplanarPoints (A, B, C, D) returns true/false, depending on whether the four points $A, B, C$, $D$ are coplanary or not.

- polarplane $(\mathrm{A}, \mathrm{S})$ returns the equation of the polar plane of point $A$ with respect to quadric $S$, that is, the equation

$$
\left[\frac{\partial(f)}{\partial\left(x_{0}\right)}\right]_{A} x_{0}+\left[\frac{\partial(f)}{\partial\left(x_{1}\right)}\right]_{A} x_{1}+\left[\frac{\partial(f)}{\partial\left(x_{2}\right)}\right]_{A} x_{2}+\left[\frac{\partial(f)}{\partial\left(x_{3}\right)}\right]_{A} x_{3}=0
$$

where $f\left(x_{0}, x_{1}, x_{2}, x_{3}\right)=0$ is the equation of quadric $S$.

Remark 2 Two points, $A$ and $B$, are said to be conjugated respect quadric $S$ if they are harmonically separated by the intersection points of line $A B$ and $S$. The locus of the conjugated points of $A$ with respect to $S$ is a plane, named polar plane of $A$ with respect to $S$.

\section{Experimenting to generate Pascal 3D configuration}

Let us begin by testing a parametric method to generate Pascal 3D configuration, inspired by the method of the proof of Proposition 1.

The quadric, the five first side-lines of the polygonal and the common point to the first side-line and its opposite, are considered as initial objects.

Given the quadric, the first five side-lines of the polygonal line are determined by its six first vertices (different two by two and laying on the quadric).

The common point, $P_{1}$, to the first side-line $A_{1} A_{2}$ and its opposite one can be left undetermined, defining it using a parameter, $\lambda_{1}$, the following way: $P_{1}=A_{1}+\lambda_{1} \cdot\left(A_{2}-A_{1}\right)$, where $\lambda_{1} \neq 0,1$, in order $P_{1}$ to be be different from $A_{1}$ and $A_{2}$.

As $P_{1}$ is the common point to $L_{1}$ and $L_{6}$, the side-line $L_{6}$ is the line $A_{6} P_{1}$, being $A_{7}$ the other intersection point of $L_{6}$ and $S$, apart from $A_{6}$.

The other vertices can be obtained iterating the process just described to determine $A_{7}$, as detailed in the Section 4.1 .

On the other hand, it is immediate to check that condition 4) of Definition 1 can be substituted by an equivalent one, but easier to check: that the four vertices $A_{i}, A_{i+1}, A_{i+5}, A_{i+2}$ are not coplanary for $i=1$, $2, \ldots, 5$.

Finally, it is also immediate to check that, if the Pascal plane does not pass through any vertex, then the following property holds: if $L_{1}, L_{2}, L_{3}$ are not coplanary, then $P_{1}, P_{2}, P_{3}$ are not collinear, and, consequently, these three points determine plane $\pi$.

\subsection{Parametric generation method}

The process to construct Pascal 2D configuration described in Proposition 1, suggests a way to generate Pascal 3D configuration that is detailed in the following Algorithm 1 (where the command of Section 3 that can be used to execute each step is detailed). 
Algorithm 1 Input: quadric $S$; vertices $A_{i}, i=1, \ldots, 6$ (with command vertex) Output: vertices $A_{7}, \ldots, A_{10}$; Pascal plane $\pi$; points $P_{1}, \ldots, P_{5}$ Steps:

1. $L_{i}:=A_{i} A_{i+1} ; i=1, \ldots, 5$ (with command line)

2. $P_{1}:=A_{1}+\lambda_{1} \cdot\left(A_{2}-A_{1}\right)$

3. $L_{6}:=A_{6} P_{1}$ (with command line)

4. $A_{7}:=L_{6} \cap S$, such that $A_{7} \neq A_{6}$ (with command newVertex)

5. $P_{2}:=A_{2}+\lambda_{2} \cdot\left(A_{3}-A_{2}\right)$

6. $L_{7}:=A_{7} P_{2}$ (with command line)

7. $A_{8}:=L_{7} \cap S$, such that $A_{8} \neq A_{7}$ (with command newVertex)

8. $P_{3}:=A_{3}+\lambda_{3} \cdot\left(A_{4}-A_{3}\right)$

9. $L_{8}:=A_{8} P_{3}$ (with command line)

10. $A_{9}:=L_{8} \cap S$, such that $A_{9} \neq A_{8}$ (with command newVertex)

11. $\pi:=$ plane $P_{1}, P_{2}, P_{3}$ (with command plane)

12. $P_{4}:=L_{4} \cap \pi \quad$ (with command intersection)

13. $L_{9}:=A_{9} P_{4}$ (with command line)

14. $A_{10}:=L_{9} \cap S$, such that $A_{10} \neq A_{9}$ (with command newVertex)

15. $L_{10}:=A_{10} A_{1}$ (with command line)

16. $P_{5}:=L_{5} \cap \pi$ (with command intersection)

17. substitute the coordinates of $P_{5}$ in the equations of $L_{10}$ (with command isPlaced)

18. solve the system of step 17 w.r.t. $\lambda_{2}, \lambda_{3}$ (with Maple solve command)

19. from among the solutions of step $18,\left\{\lambda_{2}\left(\lambda_{1}\right), \lambda_{3}\left(\lambda_{1}\right)\right\}$, choose those that verify the three conditions: 19.1 the vertices are different two by two (with command equalVertices)

$19.2 A_{i}, A_{i+1}, A_{i+5}, A_{i+2}$ are not coplanary, for $i=1,2, \ldots, 5$ (with command coplanarPoints) 19.3 the plane $\pi$ does not pass through vertices of the polygonal (with command is In).

Remark 3 In steps 12 and 16 that $P_{4} \in \pi$ and $P_{5} \in \pi$ was required. It would seem more natural to define $P_{5}=L_{5} \cap L_{10}$ and to check later that $P_{5} \in \pi$. But this would require to make sure that $L_{5}$ and $L_{10}$ intersected, what is not possible, as it depends on the values of the parameters. That is why to define $P_{5}=L_{5} \cap \pi$ and to impose later that $P_{5} \in L_{10}$ has been chosen (what is equivalent).

Example of execution of Algorithm 1 In order to simplify the calculation, a quadric and vertices, such that the coordinates of the latter have simple expressions, have been chosen. They are ellipsoid $S$, of equation $3 x_{1}^{2}+2 x_{2}^{2}+x_{3}^{2}-36 x_{0}^{2}=0$, and the closed polygonal which first six vertices are $A_{1}=(1,0,0,6)$, $A_{2}=(1,0,0,-6), A_{3}=(1,1,2,5), A_{4}=(1,2,-2,-4), A_{5}=(1,3,0,3), A_{6}=(1,-2,-2,-4)$. When executing Algorithm 1 with this input data, three solutions are obtained at Step 18.

$1^{\text {st }}$ Solution: $\left\{\lambda_{2}=0, \lambda_{3}=\frac{22}{47}\right\}$

As $\lambda_{2}=0$, obviously $P_{2}=A_{2}$ and therefore plane $\pi$ passes through vertex $A_{2}$. Moreover, substituting these values of the parameters, that $A_{8}=A_{2}$ and $A_{10}=A_{1}$ (for any value $\lambda_{1}$ ) is obtained, so the polygonal has two coincident vertices, and therefore this solution does not provide a Pascal 3D configuration in the sense of Definition 1. Moreover, plane $\pi$ also passes through vertices $A_{1}, A_{8}, A_{9}$ and $A_{10}=A_{1}$. (This will be interpreted below).

$2^{\text {nd }}$ Solution: $\left\{\lambda_{2}=\lambda_{2}, \lambda_{3}=\frac{3 \lambda_{1} \lambda_{2}-\lambda_{1}-\lambda_{2}+1}{3 \lambda_{1} \lambda_{2}+6-4 \lambda_{2}-6 \lambda_{1}}\right\}$

Substituting these values of the parameters, that $A_{5}$ and $A_{10}$ coincide is obtained, and also $P_{5}=A_{5}$ (for any values of $\lambda_{1}$ and $\lambda_{2}$ ), so the polygonal has two coincident vertices, and therefore this solution does not provide a Pascal 3D configuration in the sense of Definition 1. That $\pi$ passes through these two coincident vertices will be interpreted below.

$3^{\text {rd }}$ Solution: $\left\{\lambda_{2}=\frac{-60\left(\lambda_{1}-1\right)}{14 \lambda_{1}+55}, \lambda_{3}=\frac{74 \lambda_{1}-5}{5\left(2 \lambda_{1}+9\right)}\right\}$

Substituting these values of the parameters, the conditions of Steps 19.1, 19.2 and 19.3 of Algorithm 1 are 
verified, and therefore this solution does provide a Pascal 3D configuration in the sense of Definition 1 . The long expressions obtained for the coordinates of the four last points are omitted for the sake of brevity,, as well as those of the five points $P_{1}, P_{2}, \ldots, P_{5}$ (all depending on parameter $\lambda_{1}$ ).

Summarizing, only the $3^{\text {rd }}$ solution provides a Pascal 3D configuration in the sense of Definition 1. More precisely, in such case, a sheaf of Pascal 3D configurations depending on parameter $\lambda_{1}$ is obtained.

\subsection{Conjectures regarding Pascal 3D configurations}

In the previous example a one dimensional family of Pascal configurations depending on parameter $\lambda_{1}$ has been obtained. Consequently, for any possible value of $\lambda_{1}\left(0 \neq \lambda_{1} \neq 1\right)$, that is, for any possible position of $P_{1}$ on the side-line $L_{1}\left(A_{1} \neq P_{1} \neq A_{2}\right)$, a Pascal configuration is obtained. The result obtained induces to formulate the following conjecture.

Conjecture 1 Given a quadric $S$, the first five side-lines $L_{1}, L_{2}, L_{3}, L_{4}, L_{5}$ of a polygonal (or, what is equivalent, its first six vertices $\left.A_{1}, A_{2}, A_{3}, A_{4}, A_{5}, A_{6} \in S\right)$ and a point $P_{1}$ on $L_{1}$, such that $P_{1} \notin S, a$ Pascal $3 D$ configuration with these initial elements does exist.

On the other hand, in the example of Section 2.1 the five diagonal-lines passing through each pair of opposite vertices of the polygonal do all share the point at infinity $(0,0,0,1)$. Moreover, the polar plane of this point with respect to sphere $S$ is the Pascal plane $x_{3}=0$.

In the example of Section 2.2, the five diagonal-lines passing through each pair of opposite vertices of the polygonal do all pass through the centre of the sphere, $O$. Moreover, the polar plane of $O$ with respect to sphere $S$ is the Pascal plane $x_{0}=0$.

That leads to wonder if these properties also hold in the Pascal 3D configuration of the $3^{\text {rd }}$ example of the preceding section. In effect, in such case, the common point, $V$, of the side-lines passing through opposite vertices $A_{1} A_{6}$ and $A_{2} A_{7}$ (that can be obtained using command intersection), turns out to be $\left[-10 \lambda_{1}+5,12 \lambda_{1}, 12 \lambda_{1}, 30\right]$. This point $V$ lies on all the other side-lines passing through two opposite side-lines: $A_{3} A_{8}, A_{4} A_{9}$ and $A_{5} A_{10}$ (what can be checked with command is In). Finally, the polar plane of $V$ with respect to the ellipsoid $S$ (obtained with command polarPlane of our package) turns out to be $\left(60 \lambda_{1}-30\right) x_{0}+6 \lambda_{1} x_{1}+4 \lambda_{1} x_{2}+5 x_{3}=0$, that is the same Pascal plane $\pi$ obtained in the example of Section 4.1 for the $3^{\text {rd }}$ solution.

The same result has been reached in other examples, that have been omitted for the sake of brevity. It leads to formulate the following conjecture.

Conjecture 2 Given a Pascal configuration on a quadric S, the five lines passing through each two opposite vertices polygonal do all share a point, which polar plane with respect to $S$ is its Pascal plane.

In order to prove these conjectures, a projective reference system that allowed to choose $A_{1}, A_{2}, \ldots, A_{5}$ as the points of coordinates $(1,0,0,0),(0,1,0,0),(0,0,1,0),(0,0,0,1),(1,1,1,1)$ could be adopted, and a 4 -dimensional family of quadrics passing through those five points cold be considered, but calculations are extraordinarily complicated.

In the following section these conjectures are treated in a synthetic way, that has been reached applying the parametric generation method to concrete cases.

\section{Pascal 3D complete configuration}

Definition 2 Given a closed polygonal line with 10 vertices, $A_{1}, A_{2}, \ldots, A_{10}$ (different two by two), the five lines passing through opposite vertices of the polygonal will be named main diagonals of the polygonal, and will be denoted $R_{1}=A_{1} A_{6}, R_{2}=A_{2} A_{7}, R_{3}=A_{3} A_{8}, R_{4}=A_{4} A_{9}, R_{5}=A_{5} A_{10}$

Now, Conjecture 2 of Section 4 can be enunciated as follows: in a Pascal 3D configuration, all the main diagonals share a point, which polar plane with respect to the quadric is the Pascal plane of the configuration. To prove it we shall use the following well-known lemma. 
Lemma 1 Let $A, B, C, D$ be four points on a non-degenerated conic, $\sigma$. Then the intersection points $V=A B \cap C D$ and $P=A D \cap B C$ are harmonically separated by the intersection points of $\sigma$ and line $V P$ (that is, $V$ and $P$ are conjugated with respect conic $\sigma$-see Figure 6).

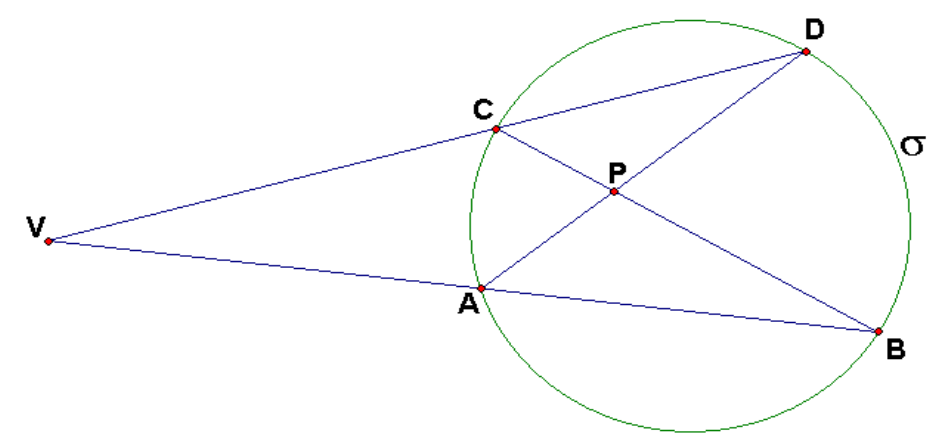

Figure 6. $V$ and $P$ are conjugated points with respect to $\sigma$.

We shall begin by proving the following result, that is somehow the reciprocal of Conjecture 2 .

Theorem 1 Let L be a closed polygonal with 10 vertices (different two by two) and side-lines different two by two, inscribed in a non-degenerated quadric, $S$. If the five main diagonals of $L$ share a point, $V$, then each side-line of $L$ intersects its opposite one, and the five intersection points of opposite side-lines, $P_{1}, \ldots$, $P_{5}$, lie on a same plane. Such plane is the polar plane of point $V$ with respect to quadric S (Figure 7).

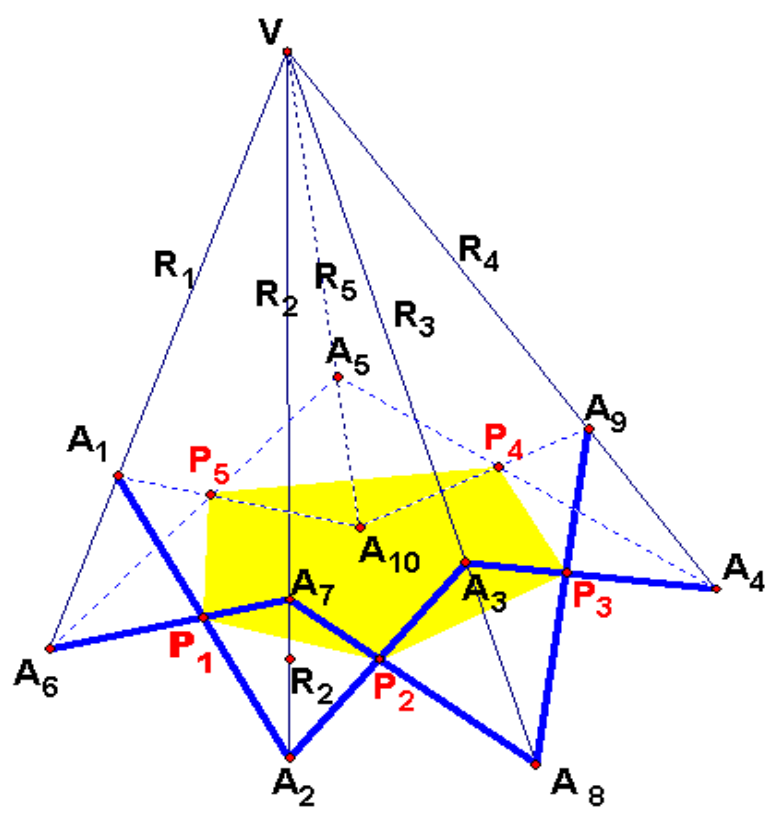

Figure 7. Concurrency of the main diagonals. 
Proof. According to the notation of Definition 2, as $R_{1}$ and $R_{2}$ do intersect, the four vertices $A_{1}, A_{6}$, $A_{2}, A_{7}$ are coplanary, and, consequently, the side-lines $L_{1}$ and $L_{6}$ are coplanary. Therefore, as $L_{1}$ and $L_{6}$ are different form each other (by hypothesis), they share exactly one point, $P_{1}$. On the other hand, as $L$ is a polygonal inscribed in $S$, vertices $A_{1}, A_{6}, A_{2}, A_{7}$ lie on $S$, and therefore they also lie on the conic $\sigma$ (intersection of $S$ with the plane through those for points). Moreover, as $P_{1}$ is the intersection point of $L_{1}=A_{1} A_{2}$ and $L_{6}=A_{6} A_{7}$ and $V$ is the intersection point of of $R_{1}=A_{1} A_{6}$ and $R_{2}=A_{2} A_{7}$, according to Lemma 1, $P_{1}$ and $V$ are conjugated with respect to conic $\sigma$, and, consequently, they are also conjugated with respect to quadric $S$.

That the other pair of opposite side-lines of the polygonal $L$ intersect, and that their respective intersection points, $P_{2}, P_{3}, P_{4}, P_{5}$, are conjugated of point $V$ with respect to quadric $S$, can be proven the same way.

Consequently, points $P_{1}, P_{2}, P_{3}, P_{4}, P_{5}$ lie on the polar plane of point $V$ with respect to quadric $S$ (that is, the locus of the conjugated points of $V$ with respect to $S$ ).

Corollary 1 Let $L$ be a closed polygonal with 10 vertices (different two by two) and side-lines different two by two, inscribed in a non-degenerated quadric, $S$. If the five main diagonals of $L$ share a point, $V$, then polygonal $L$ determines a Pascal $3 D$ configuration on $S$.

Proof. As points $P_{1}, P_{2}, P_{3}, P_{4}, P_{5}$ are coplanary, it is a Pascal 3D configuration in the sense of Definition 1.

The following result solves Conjecture 1.

Theorem 2 Given a quadric, $S$, the five first side-lines, $L_{1}, \ldots, L_{5}$, of a polygonal inscribed in $S$ (that is, which vertices lie on $S$ ) and a point $P_{1} \in L_{1}, P_{1} \notin S$, such that Condition 4 of Definition 1 is verified, then a Pascal 3D configuration with those initial elements does exist. Moreover, its five main diagonals are concurrent at a point, $V$, which polar plane with respect to $S$ is the Pascal plane of the configuration (that is, the plane containing points $P_{1}, \ldots, P_{5}$, intersection of opposite sides of the polygonal). Such configuration is unique for those initial points.

Proof. Let us suppose that quadric $S$, the six first vertices of the inscribed polygonal, $A_{1}, \ldots, A_{6}$ (or, what is equivalent, its first five side-lines $\left.L_{1}, \ldots, L_{5}\right)$ and a point, $P_{1} \in L_{1}, P_{1} \notin S$, are fixed. From these elements, the following elements are successively determined (Figure 7): side $L_{6}=A_{6} P_{1}$; vertex $A_{7}=L_{6} \cap S$ (their other intersection point, apart from $A_{6}$ ); the main diagonal $R_{1}=A_{1} A_{6}$; the main diagonal $R_{2}=A_{2} A_{7}$; point $V=R_{1} \cap R_{2}$ and the polar plane, $\pi$, of $V$ with respect to $S$. Now the following elements are also determined: lines $R_{3}=A_{3} V, R_{4}=A_{4} V, R_{5}=A_{5} V$; points $A_{8}=R_{3} \cap S$ $\left(A_{8} \neq A_{3}\right), A_{9}=R_{4} \cap S\left(A_{9} \neq A_{4}\right), A_{10}=R_{5} \cap S\left(A_{10} \neq A_{5}\right)$. According to Theorem 1 and Corollary 1 , the polygonal of vertices $A_{1}, \ldots, A_{10}$ constructed this way determines a Pascal 3D configuration, which Pascal plane is $\pi$.

We still have to prove that the Pascal 3D configuration is unique for the already mentioned initial elements. In effect, once Pascal plane, $\pi$, has been determined, the following elements are successively determined (Figure 7): side $L_{6}=A_{6} P_{1}$; vertex $A_{7}=L_{6} \cap S\left(A_{7} \neq A_{6}\right)$; point $P_{2}=L_{2} \cap \pi$; the side-line $L_{7}=A_{7} P_{2}$; vertex $A_{8}=L_{7} \cap S\left(A_{8} \neq A_{7}\right)$; point $P_{3}=L_{3} \cap \pi$; the side-line $L_{8}=A_{8} P_{3}$; vertex $A_{9}=L_{8} \cap S\left(A_{9} \neq A_{8}\right)$; point $P_{4}=L_{4} \cap \pi$; the side-line $L_{9}=A_{9} P_{4}$; vertex $A_{10}=L_{9} \cap S\left(A_{10} \neq A_{9}\right)$. And, on the other hand, for the already mentioned initial elements, the Pascal plane can not be different from plane $\pi$, determined previously. In effect, if the Pascal plane was $\pi^{\prime} \neq \pi$, then the pole of $\pi^{\prime}$ with respect to quadric $S$ would be a point $V^{\prime} \neq V$. Consequently, for the corresponding main diagonals $R_{1}^{\prime}=V^{\prime} A_{1}$ and $R_{2}^{\prime}=V^{\prime} A_{2}$, at least one of the following inequalities would be verified: $R_{1}^{\prime} \neq R_{1}$ or $R_{2}^{\prime} \neq R_{2}$, what would imply that $R_{1}^{\prime}$ either would not pass through vertex $A_{6}$ or $R_{2}^{\prime}$ would not pass through vertex $A_{7}$ (these vertices were determined in a unique way by the already mentioned initial elements). Consequently, the Pascal 3D configuration is unique for the given initial elements.

Let us observe that for the three inequalities $A_{8} \neq A_{3}, A_{9} \neq A_{4}, A_{10} \neq A_{5}$ considered in the previous proof to hold, that lines $R_{3}, R_{4}, R_{5}$ are not tangent to $S$ is required, or, what is equivalent, that $A_{3}, A_{4}, A_{5}$ 
are not contained in the cone of vertex $V$ tangent to the quadric is required. For this condition to hold, it is enough that none of those three points lie on the polar plane of $V$ with respect to $S$, that is, it is enough that they do not lie on the conic intersection of this polar plane with $S$, denoted ramification conic of $S$ with respect to $V$. In such assumption, the following result, that solves Conjecture 2, follows from Theorem 2.

Corollary 2 In a Pascal 3D configuration, all the main diagonals share a point, which polar plane with respect to the quadric is the Pascal plane of the configuration.

Proof. The point $V$ mentioned in Theorem 2 lies on the five main diagonals $R_{1}, R_{2}, R_{3}, R_{4}, R_{5}$.

Now, Corollary 2 allows to complete the configuration considered in Definition 1, as detailed afterwards.

Definition 3 The geometric configuration consisting of the elements considered in Definition 1, together with the five main diagonals of the polygonal and its point of concurrency, will be referred to as Pascal 3D complete configuration (see Figure 7). Point V, of concurrency of the five main diagonals, will be named Pascal centre of the configuration hereinafter.

\section{Generating the complete configuration}

The results of Section 5 suggest a simple method to generate a Complete Pascal 3D configuration, based on the concurrency of the five main diagonals of the 10 side closed polygonal inscribed in the non-degenerated quadric.

We shall begin by considering the same initial elements as in Section 4, that is: the quadric, the five first side-lines of the polygonal and the common point of the first side-line and its opposite one (determined by a parameter, $\lambda_{1}$, as in Section 4 ).

We shall describe another method later, considering as initial elements: the quadric, the Pascal centre and the four first side-lines of the polygonal (or, what is equivalent, the first five vertices of the polygonal).

On the other hand, that Condition 4 of Definition 1 can be substituted by an equivalent one, but much simpler to check (that the four points $V, A_{i}, A_{i+1}, A_{i+2}$, for $i=1,2, \ldots, 5$, are not coplanary), can be checked immediately.

\subsection{Generation based on the concurrency of the main diagonals}

The process described in the proof of Theorem 2 suggests a way to generate the Pascal 3D complete configuration, that is detailed in Algorithm 2 below (where the command of Section 3 that can be used to execute each step is detailed).

Algorithm 2 Input: quadric $S$; vertices $A_{i}, i=1, \ldots, 6$ (with command vertex) Output: vertices $A_{7}, A_{8}, A_{9}, A_{10}$; Pascal centre $V$; Pascal plane $\pi$; points $P_{1}, \ldots, P_{5}$ Steps:

1. $L_{i}:=A_{i} A_{i+1} ; i=1, \ldots, 5$ (with command line)

2. $P_{1}:=A_{1}+\lambda_{1} \cdot\left(A_{2}-A_{1}\right)$

3. $L_{6}:=A_{6} P_{1}$ (with command line)

4. $A_{7}:=L_{6} \cap S$, such that $A_{7} \neq A_{6}$ (with command newVertex)

5. $R_{1}:=A_{1} A_{6}$ (with command line)

6. $R_{2}:=A_{2} A_{7}$ (with command line)

7. $V:=R_{1} \cap R_{2}$ (with command intersection)

8. $R_{i}:=A_{i} V ; i=3,4,5$ (with command line)

9. $A_{i+5}:=R_{i} \cap S$, such that $A_{i+5} \neq A_{i}, i=3,4,5 \quad$ (with command newVertex)

10. $L_{i}:=A_{i} A_{i+1} ; i=7,8,9$ (with command line)

11. $L_{10}:=A_{10} A_{1}$ (with command line ) 
12. $\pi:=$ polar plane of $V$ with respect to $S$ (with command polarplane)

13. $P_{i}:=L_{i} \cap \pi ; i=2,3,4,5$ (with command intersection)

14. check that it is is a complete Pascal configuration by checking that:

14.1 the vertices are different two by two (with command equalVertices)

$14.2 \mathrm{~V}, A_{i}, A_{i+1}, A_{i+2}$ are not coplanary, for $i=1,2, \ldots, 5$ (with command coplanarPoints)

14.3 plane $\pi$ does not pass through vertices $A_{i} ; i=3,4,5$ (with command is In).

Remark 4 It is enough that $A_{i} \notin \pi ; i=3,4,5$ to assure that $A_{i+5} \notin \pi ; i=3,4,5$, because $A_{i+5}$ and $A_{i}$ are harmonically separated with respect to $V$ and $P_{i}$. On the other hand, from Theorem 2 and Corollary 2 , it follows that $P_{1}$ lies on $\pi$ and that $P_{2}, P_{3}, P_{4}, P_{5}$ lie on $L_{7}, L_{8}, L_{9}, L_{10}$ (what can now also be experimentally checked using command is In).

Example of execution of Algorithm 2 In order to simplify the calculations, we shall consider as initial elements a quadric and vertices which coordinates have simple expressions. They are the hyperboloid $S$ of equation $4 x_{1}^{2}+9 x_{2}^{2}-x_{3}^{2}-36 x_{0}^{2}=0$ and the vertices $A_{1}=(1,5,0,8), A_{2}=(1,5,0,-8), A_{3}=(1,2,2,4)$, $A_{4}=(1,2,-2,-4), A_{5}=(1,6,2,12), A_{6}=(1,3,0,0)$. The execution of Algorithm 2, for these initial data is detailed afterwards.

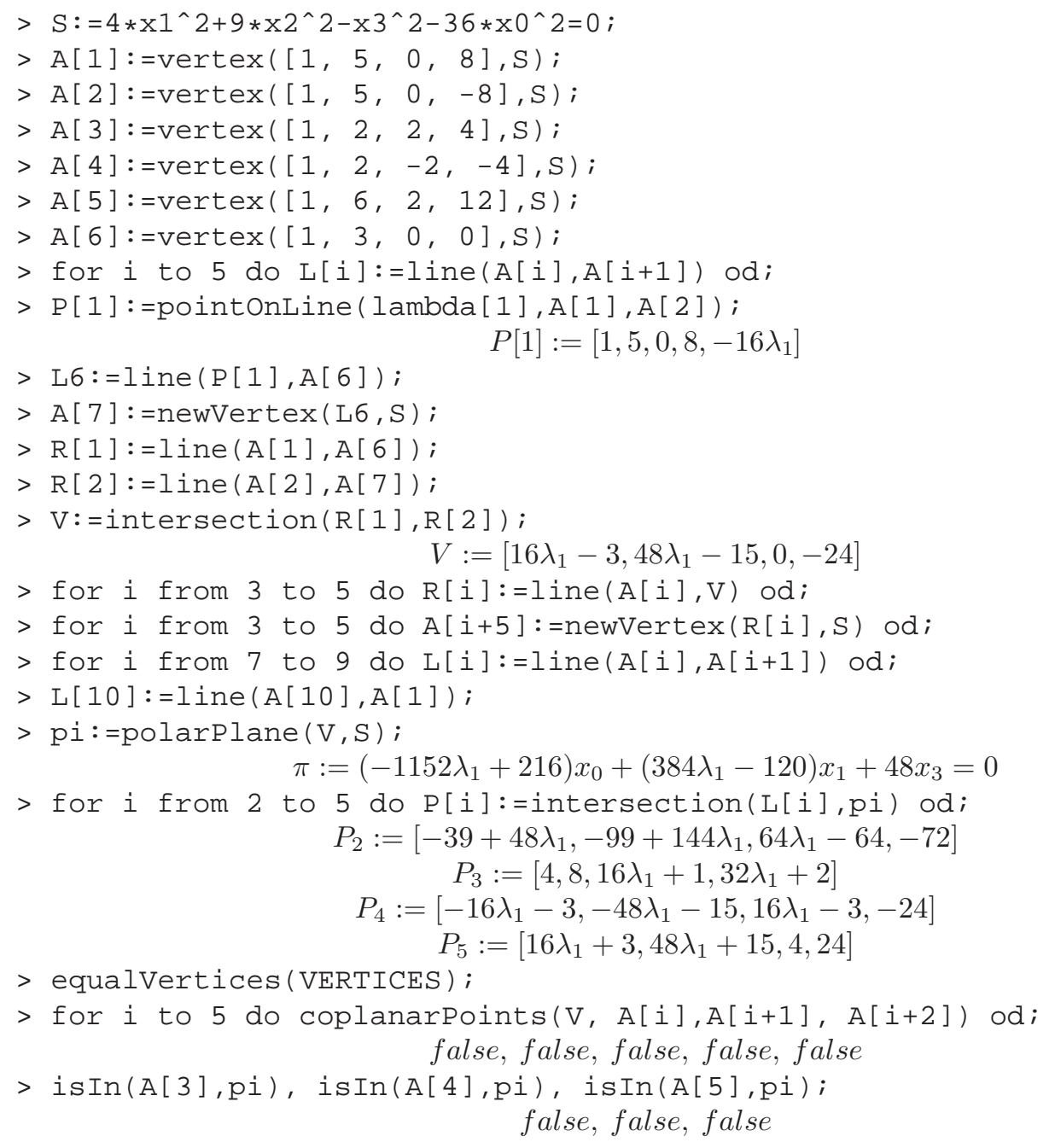


Remark 5 Comparing the parametric method of Section 4 with this method based on the concurrency of the main diagonals, the advantage of the latter is clear (it operates with much shorter expressions, involving just one parameter instead of three).

\subsection{Generation from Pascal point}

The generation process is even more simplified when considering as initial elements the quadric $S$, the Pascal centre $V$ and the five first vertices $A_{1}, A_{2}, A_{3}, A_{4}, A_{5}$ (that must not lie on the polar plane of $V$ with respect to $S$, so that they do not lie on the ramification conic of $S$ with respect to $V$ ). The process is detailed in the following Algorithm 3 (where the command of Section 3 that can be used to execute each step is detailed).

Algorithm 3 Input: quadric $S$; Pascal centre $V(V \notin S)$; vertices $A_{i}\left(A_{i} \neq V\right), i=1, \ldots, 5$ Output: vertices $A_{6}, A_{7}, A_{8}, A_{9}, A_{10}$; points $P_{1}, \ldots, P_{5}$; Pascal plane $\pi$

Steps:

1. $R_{i}:=A_{i} V ; i=1, \ldots, 5$ (with command line)

2. $A_{i+5}:=R_{i} \cap S$, such that $A_{i+5} \neq A_{i}$ (with command newVertex)

3. $A_{11}:=A_{1}$ (in order to obtain $L_{10}$ in a briefer way)

4. $L_{i}:=A_{i} A_{i+1} ; i=1, \ldots, 10$ (with command line)

5. $P_{i}:=L_{i} \cap L_{i+5} ; i=1, \ldots, 5$ (with command intersection)

6. $\pi:=$ polar plane of $V$ with respect to $S$ (with command polarPlane)

7. Check that it is complete Pascal 3D configuration, by verifying:

7.1 the vertices are different two by two (with command equalVertices)

$7.2 \mathrm{~V}, A_{i}, A_{i+1}, A_{i+2}$ are not coplanary for $i=1,2, \ldots, 5$ (with command coplanarPoints)

7.3 plane $\pi$ passes through points $P_{i} ; i=1, \ldots, 5$ (with command is In).

Remark 6 The plane $\pi$ can also be obtained as the plane through three non-collinear points from among the five points $P_{1}, P_{2}, \ldots, P_{5}$.

Example of execution of Algorithm 3 In order to simplify the calculations, we shall consider as initial points a quadric and vertices which coordinates have simple expressions. They are the paraboloid $S$ of equation $x_{1}^{2}+3 x_{2}^{2}+4 x_{0} x_{3}=0$, the Pascal point $V=(1,2,1,12)$ and the closed polygonal which first five vertices are $A_{1}=(1,0,2,-3), A_{2}=(1,1,1,-1), A_{3}=(1,2,0,-1), A_{4}=(1,1,-1,-1)$, $A_{5}=(1,0,-2,-3)$.

When executing Algorithm 3 with these data, the following geometric objects are successively obtained: the main diagonals of the polygonal; the five remaining vertices, of coordinates $A_{6}=(7,-96,62,-741)$, $A_{7}=(1,-53,1,-703), A_{8}=(3,6,-52,-679), A_{9}=(13,-29,-97,-559), A_{10}=(31,-48,-134$, -453) (the ten vertices are automatically allocated in the global variable VERTICES); the ten side-lines of the polygonal; the five intersection points of opposite side-lines, of coordinates $P_{1}=(1,-8,10,-19)$, $P_{2}=(-1,-28,26,1), P_{3}=(-5,16,26,5), P_{4}=(3,-4,-10,-17), P_{5}=(-117,96,158,1071)$ and the Pascal plane, of equation $24 x_{0}+2 x_{1}+3 x_{2}+2 x_{3}=0$. Finally, that the steps 7.1, 7.2 and 7.3 of Algorithm 3 are verified is checked.

Remark 7 In this example, the four first points $P_{i}$ of intersection of opposite side-lines of the close polygonal are exterior to the corresponding sides (segments), as can be easily checked (the fifth can not be exterior, as the polygonal is closed).

\section{Conclusions}

In order to extend Pascal 2D to 3D in a natural way, substituting conic by quadric, the six sides closed polygonal has been substituted by a ten sides one, because $10-1$ points determine a quadric, the same way 
as $6-1$ points determine a conic. But in the 3D case, that each side-line of the polygonal intersects its opposite one has to be checked. In case this holds, those five intersection points lie on a plane (that we have named Pascal plane), the same way as in the 2D case the intersection points of opposite side-lines lie on a line (Pascal line).

Once this 3D configuration is defined, simple particular cases have been considered (Section 2), that have suggested ideas to advance in the problem. But, when trying to analytically treat not so simple cases, long expressions arise, what has made advisory to use a computer algebra system (Maple), where an ad-hoc package has been implemented (it is described in Section 3). Using its commands, a parametric generation method to generate the 3D configuration in an analytic way from the following initial elements: the quadric, the first five side-lines and the common point to the first side-line and its opposite one, has been applied (Section 4). With this method, that in all the examples tested the five main diagonals of the polygonal share a point, which polar plane with respect the quadric is the Pascal plane, has been checked. These observations have lead to conjecture their general validity, that was solved in a synthetic way in Section 5. The common point to all the main diagonals have been denoted Pascal centre. These new elements that complete our Pascal 3D configuration have allowed to simplify the construction process of the configuration in Section 6.

We finish comparing Pascal 2D classic configuration with the 3D one developed in this article. In the first one, three consecutive sides and two intersection points of opposite sides (that is, Pascal line), can be freely chosen. Meanwhile, in our extension to 3D the five consecutive sides can be freely chosen, but only one of the intersection points of opposite sides can be freely chosen. Therefore, in both cases half the side-lines can be freely chosen, three out of six in the 2D case and five out of ten in the 3D case. But, meanwhile in the 2D case two points in the Pascal line can be freely chosen, in the 3D case only one point on the Pascal plane can be freely chosen. This loss of degrees of freedom is motivated by the need to assure that in 3D the opposite side-lines of the polygonal do intersect.

Acknowledgement. This work was partially supported by the research projects MTM2004-03175 (Ministerio de Educación y Ciencia, Spain), UCM2006-910563 (Comunidad de Madrid - Universidad Complutense de Madrid, research group ACEIA) and TIN2006-06190 (Ministerio de Educación y Ciencia, Spain).

\section{References}

[1] Bottema, O., (1955). A Generalization of Pascal's Theorem, Duke Math. J., 22, 123-127.

[2] Chasles, M., (1889). Aperçu Historique sur l'Origine et le Développment des Méthodes en Géométrie, Gauthier-Villars, (3rd. ed.), Paris (p. 400).

[3] Clifford, W. K., (1864). Analogues of Pascal's Theorem Quart. J. Math., 23, 72-79.

[4] Court, N. A., (1953). Pascal's theorem in space. Duke Math. J., 20, 417-421.

[5] Fernández-Biarge, J., (2008). Una generalización del teorema de Pascal al espacio de tres dimensiones. Bol. Soc. Puig Adam Prof. Mat., 79, 21-25.

[6] Fox, C., (1958). The Pascal line and its generalizations. The American Math. Monthly, 65/3, 185-190.

[7] Roanes-Macías, E. And Roanes-Lozano, E., (2001). Automatic determination of geometric loci. 3DExtension of Simson-Steiner Theorem. In: J. A. Campbell and E. Roanes-Lozano (eds.), Artificial Intelligence and Symbolic Computation. International Conference AISC 2000. Springer-Verlag LNCS 1930, BerlinHeidelberg-New York (157-173).

[8] Roanes-Macías, E. And Roanes-Lozano, E., (2002). Geometric determination of the spheres that are tangent to four given ones. In: P. M. A. Sloot, J. J. Dongarra, C. J. K. Tan and A. G. Hoekstra (eds.), Computational Science - ICCS 2002. Springer-Verlag LNCS 2330, Berlin-Heidelberg-New York (52-61). 
[9] Roanes-Macías, E. And Roanes-Lozano, E., (2004). A Completion of Hypotheses Method for 3DGeometry. 3D-Extensions of Ceva and Menelaus Theorems. In: J. M. Diaz Bánez, A. Márquez and J. R. Portillo (eds.), Proceedings of $20^{\text {th }}$ European Workshop on Computational Geometry. Universidad de Sevilla, Seville (85-88).

[10] Roanes-Macías, E. And Roanes-Lozano, E., (2004). A method for outlining 3D-problems in order to study them mechanically. Application to prove the 3D-version of Desargues Theorem. In: L. González Vega and T. Tecio (eds.), Proceedings of EACA 2004. Univ. of Cantabria, Santander (237-242).

[11] Roanes-Macías, E., Roanes-Lozano, E. And Fernández-Biarge, J., (2007). Extensión a 3D del teorema de Desargues, sustituyendo triángulos por tetraedros. Bol. Soc. Puig Adam Prof. Mat., 75 13-28.

[12] Roanes-Macías, E. And Roanes-Lozano, E., (2007). 3D-extension of Steiner's chain problem. Mat. Comp. Mod., 45, 137-148.

[13] Roanes-Macías, E. And Roanes-Lozano, E., (2007). A Maple Package for Automatic Theorem Proving and Discovery in 3D-Geometry. In: F. Botana and T. Recio (eds), Automated Deduction in Geometry. 6th International Workshop, ADG 2006. Springer-Verlag LNAI 4869, Berlin-Heidelberg-New York (171-188).

[14] Roanes-Macías, E., Roanes-Lozano, E. And Fernández-Biarge, J., (2008). Extensión natural a 3D del teorema de Pappus y su configuración completa. Bol. Soc. Puig Adam Prof. Mat., 80, 38-56.

[15] Salmon, G., (1912). A treatise on the Analytic Geometry of Three Dimensions, Longmans, Green and Company, London.

\section{Eugenio Roanes-Macías}

Associate Professor of Algebra (Ret.)

Algebra Dept.

School of Education

Universidad Complutense de Madrid

\section{Julio Fernández-Biarge}

Emeritus Professor of Applied Mathematics

E.T.S.I. Navales

Universidad Politécnica de Madrid

\section{Eugenio Roanes-Lozano}

Associate Professor of Algebra

Algebra Dept.

School of Education

Universidad Complutense de Madrid 